\title{
Pancreatitis caused by tigecycline: A rare case report
}

\author{
Yinyin Guo ${ }^{1}$, Laiyuan $\mathrm{Li}^{2}$, Xia Song ${ }^{1}$ and Hui Zhao ${ }^{1 *}$ \\ ${ }^{1}$ Lanzhou University Second Hospital, China \\ ${ }^{2}$ Department of Anorectal Surgery, Gansu Provincial Hospital, China
}

\begin{abstract}
Tigecycline is a broad-spectrum antibiotic, obtained by modifying earlier tetracycline, to prevent resistance mechanisms and is effective against many multidrugresistant organisms [1,2]. According to the naranjo adverse drug reaction probability scale, tigacycline can cause pancreatitis during treatment [3]. Clinicians monitor patients for signs and symptoms related to amylase levels during treatment with tigecycline. We here report an adverse effect of tigecycline with a case of acute pancreatitis, which occurred on the 8th day of tigecycline administration and improved after its withdrawal.
\end{abstract}

\section{Introduction}

Tigecycline was approved on the market in 2005 by the US Food and Drug Administration (FDA). It is a broad-spectrum antibiotic that shows remarkable efficacy against many multiple drug-resistant (MDR) pathogens. The common side effects of tigecycline include nausea and vomiting. With the increasing detection rate of drug-resistant bacteria, the application of tigecycline increased significantly. Therefore, tigecycline-associated adverse reactions are increasing. In this report, we present a patient who was diagnosed with $\operatorname{IgA}$ nephropathy and pneumonia and presented with acute pancreatitis after the use of tigecycline.

\section{Case presentation}

On May 16, 2018, a 44-year-old male patient was admitted to our hospital with cough, expectoration and fever. He was diagnosed with community-acquired pneumonia. Then, the patient received moxifloxacin for the treatment of the infection. The patient had a history of IgA nephropathy and the long-term administration of hormones and cyclophosphamide. On the 7th day of hospitalization, the patient's infection was exacerbated, with an elevated white blood cell count (WBC) of $18.9^{\star} 10^{9} / \mathrm{L}$, a procalcitonin (PCT) level of 1.920 $\mathrm{ng} / \mathrm{mL}$ and a high temperature of $39.3^{\circ} \mathrm{C}$. He was transferred to the intensive care unit (ICU) and started on mechanical ventilation due to severe pneumonia on May 23, 2018. According to his clinical symptoms and laboratory data, intravenous moxifloxacin, sulfamethoxazole and ganciclovir were administered. Continuous renal replacement therapy (CRRT) was performed because of his high blood creatinine level $(540.8 \mu \mathrm{mol} / \mathrm{L})$ and oliguria.

On the 2nd day after admission to the ICU, the patient's temperature gradually increased, and his clinical symptoms became significantly worse. We adjusted the anti-infection programme. The patient was treated with cefoperazone, sulbactam, sulfamethoxazole and echinocandin. After 3 days of the anti-infection programme, his clinical symptoms improved. X-ray showed that his pulmonary infection was improved.

Unfortunately, the patient's oxygen saturation level dropped to $70 \%$ on his 8 th day in the ICU. The blood culture and sputum culture were negative. Multiple drug-resistant Acinetobacter baumannii is one of the main pathogens that cause infections in the ICU. We adjusted the treatment plan to address an infection with Acinetobacter baumannii. The doctor added tigecycline to the treatment programme. Tigecycline was administered intravenously, with an initial dose of $100 \mathrm{mg}$ and subsequent doses of $50 \mathrm{mg}$ administered every $12 \mathrm{~h}$. After 7 days of tigecycline treatment, the infection improved significantly, and the highest temperature was significantly lower than before. The patient initially tolerated this medicine without complaints of nausea or vomiting. However, physical examination found moderate tenderness in the upper abdomen. Laboratory analyses showed a marked increase in the level of amylase to $1250 \mathrm{U} / \mathrm{L}$. Computed tomography (CT) scans suggested acute edema pancreatitis (Figure 1). Shortly after tigecycline discontinuation and symptomatic treatment, the patient's symptoms gradually improved. The patient's amylase level returned to $168 \mathrm{U} / \mathrm{L}$ on the 21st day following the discontinuation of tigecycline (Figure 2). Then, the patient returned to the nephrology department for treatment. We ordered the patient to go on a low-fat diet.

\section{Discussion}

Tigecycline is a glycylcycline with a broad spectrum of antibacterial activity [4]. Its indications include skin and soft tissue infections, complex abdominal infections, and community-acquired pneumonia in adults [5]. We do not recommend it for hospitalacquired pneumonia because of the high mortality rate [6]. In this case, the rationality of using tigecycline empirically for the treatment of severe pneumonia without specific aetiological identification was questionable.

At present, the detection rate of multi-drug resistant bacteria is increasing annually. Thus, tigecycline is a better choice than many other

${ }^{\star}$ Correspondence to: Hui Zhao, Department of Pharmacy, Lanzhou University Second Hospital, 82 Cuiyingmen, Lanzhou, China. Email: 1deyzh@163.com

Key words: tigecycline, pancreatitis, case report

Received: September 27, 2019; Accepted: October 04, 2019; Published: October 07, 2019 


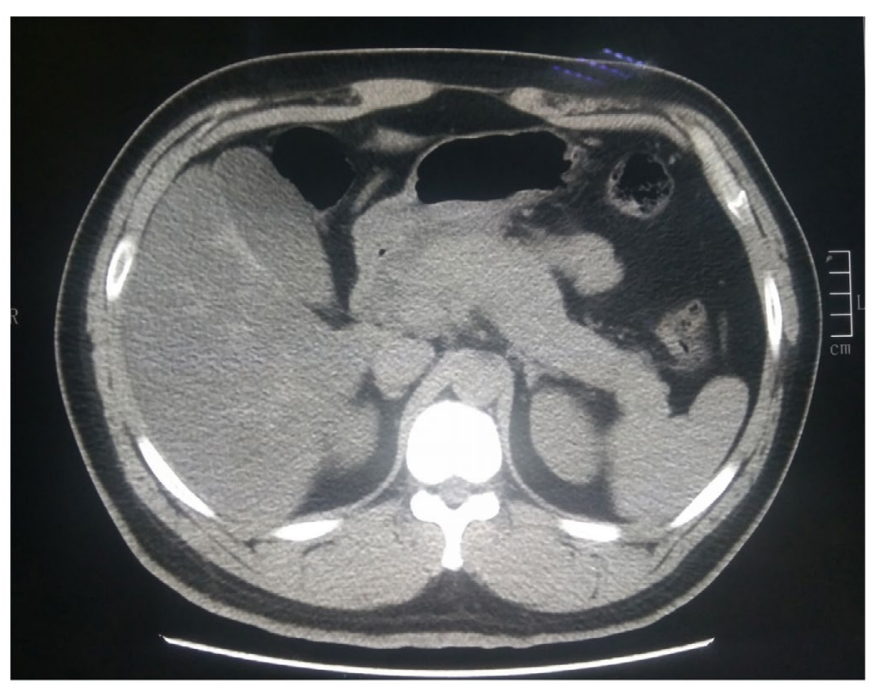

Figure 1. CT showing signs of acute edema pancreatitis

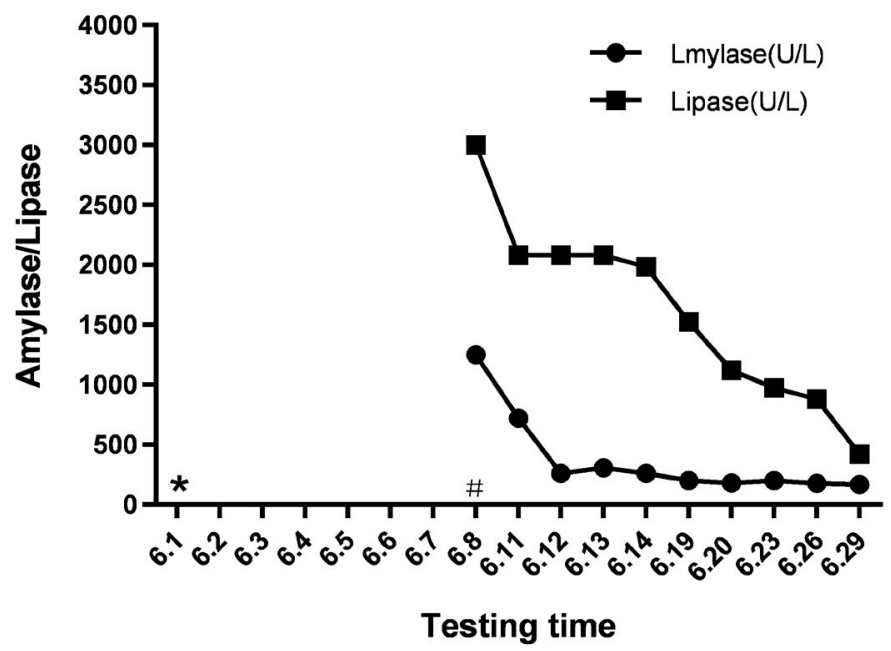

Figure 2. Amylase and lipase concentrations following initiation and withdrawal of tigecycline

("Started tigecycline, *Stopped tigecycline)

antibiotics [7]. However, we tend to ignore the severe adverse reactions. The new version of the guidelines has added information regarding the fact that the drug can cause coagulation dysfunction, cholangitis and pancreatitis. In fact, many medications, such as ceftriaxone, isoniazid, metronidazole, pentamidine and tetracycline, can also cause pancreatitis [8]. The overall incidence of drug-induced pancreatitis is $0.1-2 \%$ [9]. Tigecycline-induced pancreatitis is still considered a rare phenomenon and has only been described in a few studies. It arises in less than $1 \%$ of all patients treated with tigecycline [10]. However, tigecycline can cause an increase in the level of amylase. One phase III randomized double-blinded trial demonstrated a more frequent elevation of the amylase level in patients administered tigecycline $(9 / 292,3.1 \%)$ than in those administered vancomycin-aztreonam $(3 / 281,1.1 \%)$ [11]. In general, after the withdrawal of the drug, the symptoms improve. However, the time to the recovery of pancreatic enzyme levels differs. Okon E, et al [12]. showed that tigecyclineinduced pancreatitis is more common in women than in men. There was no plausible explanation given for this finding.
The exact mechanism of tigecycline-induced pancreatitis is still unknown. Tigecycline is a derivate of minocycline. It causes adverse reactions that are similar to those associated with minocycline. Only a few studies have shown that the concentration of minocycline in the bile is much higher than that in the peripheral blood, which is the cause of pancreatitis [13]. Similarly, after a single $100 \mathrm{mg}$ dose of tigecycline was administered, the concentrations of tigecycline in bile and the peripheral blood reached $75.2 \mathrm{mg} / \mathrm{L}$ (median) and 0.112 mg/L (median), respectively [14]. Furthermore, tetracycline can induce hypertriglyceridaemia, which is associated with pancreatitis [15]. Third, the mechanism may involve the formation of toxic metabolites.

\section{Conclusions}

Physicians should pay attention to clinical symptoms, clinical signs and the serum amylase concentration to monitor the development of pancreatitis. If necessary, abdominal CT images should be taken regularly after the administration of tigecycline.

\section{Acknowledgments}

The authors thank the patients for allowing us to publish these case reports.

\section{Disclosure statement}

The authors declare no conflict of interest.

\section{References}

1. Davido B, Shourick J, Makhloufi S (2016) True incidence of tigecycline-induced pancreatitis: how many cases are we missing? J Antimicrob Chemother 71: 2994-2995. [Crossref]

2. Park GE, Kang CI, Wi YM (2015) Case-control study of the risk factors for acquisition of Pseudomonas and Proteus species during tigecycline therapy. Antimicrob Agents Chemother 59: 5830-5833. [Crossref]

3. Naranjo CA, Busto U, Sellers EM (1981) A method for estimating the probability of adverse drug reactions. Clin Pharmacol Ther 30: 239-245. [Crossref]

4. Rose WE, Rybak MJ (2006) Tigecycline: first of a new class of antimicrobial agents Pharmacotherapy 26: 1099-1110. [Crossref]

5. Falagas ME, Metaxas EI (2009) Tigecycline for the treatment of patients with community-acquired pneumonia requiring hospitalization. Expert Rev Anti Infect Ther 7: 913-923. [Crossref]

6. https://www.fda.gov/drugs/drug-safety-and-availability/2010-drug-safetycommunications.

7. Poulakou G, Kontopidou FV, Paramythiotou E (2009) Tigecycline in the treatment of infections from multi-drug resistant gram-negative pathogens. J Infect 58: 273-284. [Crossref]

8. Hung WY, Abreu Lanfranco O (2014) Contemporary review of drug-induced pancreatitis: A different perspective. World J Gastrointest Pathophysiol 5: 405-415. [Crossref]

9. Balani AR, Grendell JH (2008) Drug-induced pancreatitis : incidence, management and prevention. Drug Saf 31: 823-837. [Crossref]

10. McGovern PC, Wible M, Korth-Bradley JM (2014) Pancreatitis in tigecycline Phase 3 and 4 clinical studies. J Antimicrob Chemother 69: 773-778. [Crossref]

11. Sacchidanand S, Penn RL, Embil JM (2005) Efficacy and safety of tigecycline monotherapy compared with vancomycin plus aztreonam in patients with complicated skin and skin structure infections: Results from a phase 3, randomized, double-blind trial. Int J Infect Dis 9: 251-261. [Crossref]

12. Okon E, Engell C, van Manen R (2013) Tigecycline-related pancreatitis: a review of spontaneous adverse event reports. Pharmacotherapy 33: 63-68. [Crossref]

13. Macdonald H, Kelly RG, Allen ES (1973) Pharmacokinetic studies on minocycline in man. Clin Pharmacol Ther 14: 852-861. [Crossref] 
14. Hung WY, Kogelman L, Volpe, G (2009) Tigecycline-induced acute pancreatitis: case report and literature review. Int J Antimicrob Agents 34: 486-489. [Crossref]
15. Lindberg DA (2009) Acute pancreatitis and hypertriglyceridemia. Gastroenterol Nurs 32: $75-82$.

Copyright: (C2019 Guo Y. This is an open-access article distributed under the terms of the Creative Commons Attribution License, which permits unrestricted use, distribution, and reproduction in any medium, provided the original author and source are credited. 